\title{
Probable Late Congenital Syphilis Presenting as Clutton Joints
}

\author{
Major J B L Kabuubi RAMC \\ Consultant Physician \\ Princess Mary's RAF Hospital, Akrotiri, BFPO 57
}

SUMMARY: A patient who presented with recurrent bilateral knee effusions was found to have positive serum syphilis serology. She was treated with oral erythromycin to a total of $\mathbf{4 0}$ grams and intra-articular injection of steroids in the right knee joint. Late congenital syphilitic arthritis is discussed with a review of the literature.

\section{Case Report}

When first seen in July 1987, a 23 year old obese Fijian female cleaner, married to a serviceman, had been living in Europe since 1984. She was complaining of spontaneous pain in both knees for 8 months, worse on climbing stairs and at night, the right more than left, and swelling of these joints for 3 months. She gave no history of constitutional symptoms and no relevant past medical history.

On examination she had no signs of congenital syphilis. All her teeth were intact. A full ophthalmic examination was normal. The audiogram showed conduction deafness in the left ear. There were no signs to suggest previous yaws infection. She had no mucocutaneous lesions and no lymphadenopathy. Routine and gonococcal microbiological cultures of the genital swabs were negative. She had tense, non tender, bilateral knee effusions but no signs of acute joint inflammation. Aspiration of both knee joints produced a clear straw coloured serous synovial fluid containing a few white blood cells on microscopy; no further biochemical or microbiological examination of the fluid was done. The Rapid Plasma Reagin (RPR) titre was 1:2. The Serum Treponema Pallidum Haemagglutination test (TPHA) was positive to a titre of 1:5120 and the Immunofluorescent Treponema Absorption test (FTA) was positive IgG, but negative IgM. Sedimentation rate was $23 \mathrm{~mm}$ in the first hour. Serum Latex fixation test for rheumatoid arthritis, Fluorescent test for antinuclear antibody and DNA binding were negative. Cerebrospinal fluid examination was normal. X-rays of chest, knees, pelvis and long bones were normal. Because of a history of allergy to penicillin she was given an oral course of erythromycin $500 \mathrm{mg}$ four times a day for twenty days. She developed no Jarisch-Herxheimer reaction.

Three months after her initial presentation she had a recurrence of the bilateral knee effusions, which were aspirated ( 23 millilitres of clear straw coloured fluid from right knee but none from the left). She had intraarticular injection $10 \mathrm{mgms}$ of triamcinolone into the right knee joint. The microscopy of the synovial fluid showed 140 red blood cells and 210 white blood cells, but the differential count was not done. No crystals were seen in the synovial fluid, and routine gonococcal and acid fast microbiological cultures of the genital swabs and synovial fluid were sterile. The synovial fluid latex fixation test for rheumatoid arthritis was negative and the glucose was $6 \mathrm{mmol} / \mathrm{L}$. The synovial fluid TPHA was positive to a titre of 1:2560 and the FTA was positive IgG and negative IgM.

When reviewed 8 months after treatment with erythromycin and 5 months after intra-articular injection of steroids she had had no recurrence of the knee effusions. All other joints were normal. Her only complaint was aching of both knees on prolonged walking, which was thought to be due to obesity. The $\mathrm{X}$-rays of both knees were normal. The Rapid Plasma Reagin titre remained 1:2. The serum TPHA was stilt positive to a titre of 1:1280 and the FTA was also positive (positive IgG and negative IGM).

\section{Family and Medical History}

She was born in Fiji and had normal physical an $\Phi$ mental development. Her mother had repeate $\$$ miscarriages and is still alive and well, living in Fiji. A the age of 50 years of age her father developed paralys of the left leg, then became paraplegic and senile and died in a psychiatric hospital a few months afterwards. She is one of five children; the second youngest died at birth and one sister died aged 17 years. The cause of these deaths is unknown. The patient reported did not have any relevant past medical history and is nulliparous.

Clinical examination of her husband was normal and his syphilitic serology was negative.

\section{Discussion}

Acquired syphilis is now a rare disease in the United Kingdom. Even in the mobile military population the disease is still quite rare. There were only two cases in BAOR during 1986 and 1987: one case of primary syphilis acquired in Berlin and the above case. Congenital syphilis is equally uncommon. Syphilitic Joint Disease (SJD) is rare but the diagnosis can be missed if it is not looked for. The largest series described in the literature is from India ${ }^{1}$ where out of 3918 patients attending the Rheumatology Clinic, 379 had positive serology and 83 were considered to have SJD. Most authors describe case histories of less than 10 patients. SJD may be either congenital or acquired. 


\section{a. Congenital SJD}

(i) Acute epiphysitis or osteochondritis in the juxta epiphyseal region during the first few weeks of life commonly affects the upper portion of the humerus leading to breakdown of cartilage (Parrot's pseudoparalysis) ${ }^{2}$.

(ii) In 1886 Clutton described bilateral hydroarthrosis of the knees in 11 patients aged between 6 and $16^{3}$, but this is now known to occur at any age up to 35 . It is mainly synovitis or peri-synovitis as there is no radiological bone damage.

\section{b. Acquired SJD}

Direct invasion of the joints by Treponema pallidum is not common, but polyarthralgia/arthritis resembling rheumatic fever during the secondary stage has been described. In Europe neuropathic joint disease is likely to be due causes other than tertiary syphilis, but 5 to $10 \%$ of patients with tabes dorsalis have neuropathic joint disease ${ }^{2}$. There may still be a few cases of syphilitic neuropathic joint disease (Charcot Joint) in the UK acquired before the 1939-45 war or abroad.

Although the patient reported had no symptoms or signs suggestive of congenital/acquired syphilis, luetic serology was done because of high index of suspicion. The diagnosis of congenital syphilis depends on the family history, other clinical and radiological manifestations of congenital syphilis and therapeutic response to anti-syphilitic drugs ${ }^{4}$. The family history of repeated maternal abortions is suggestive of luetic infection. The mother is still alive living in Fiji, therefore could not be tested. The cause of paralysis, dementia and early death of the father cannot be ascertained but luetic infection is a possibility. A reagin titre of less than $1: 8$ shows that a patient has not got active/latent syphilis or yaws. The RPR remained consistent at a titre of $1: 2$ during the time of observation. The positive serum FTA Absorption IgG and negative IgM confirms inactive infection. The positive synovial fluid syphilis serology could be due to ultrafiltration action into the synovial fluid. Synovial membrane biopsy was considered but was not done because of technical difficulties. Reginato ${ }^{5}$ analysed synovial fluid and synovial membrane biopsy both by light and electron microscopy. Polymorphonuclear and mononuclear leucocytosis was found in equal numbers of patients. Light microscopy of the synovial membrane showed lymphocytes, plasma cells and polymorphonuclear infiltration in deeper layers but no vasculitis. Electron microscopy showed treponeme-like bodies in the synovial membrane supporting direct invasion by the spirochaetes. Argen ${ }^{6}$ described a negress age 19 with Clutton joints, keratitis and periostitis. The synovial membrane biopsy report on this patient showed oedema and increased vascularity with proliferation of superficial cells consistent with chronic inflammation. Despite the above electron microscopy studies the pathogenesis of Clutton joints is considered to be autoimmune ${ }^{7}$.
Clutton joints presents with spontaneous symmetrical hydro-arthrosis of large joints usually the knees, elbows and rarely ankles but may be precipitated by trauma ${ }^{4,6,7}$. $\frac{3}{8}$ Both sides may be affected at the same time or separated $\stackrel{\varnothing}{\varnothing}$ by months or years ${ }^{4,6,7}$. The joints are usually painless $?$ with no signs of acute inflammation but may be painful, hot and even tender. The patient may or may not be feverish. X-rays of Clutton joints show effusion with $\overrightarrow{\vec{F}}$ increased joint space and soft tissue swelling (synovitis/ $\overrightarrow{0}$ perisynovitis) without bone destruction, but in $\frac{\mathrm{C}}{\sigma}$ neuropathic joints and osseous form of gummatous $\frac{\bar{\sigma}}{\bar{D}}$ arthritis there is erosion of articular cartilage with $\overrightarrow{\widetilde{D}}$ destruction of joint surfaces ${ }^{4,7}$. The patient's X-rays of $\square$ both knees have remained radiologically normal on throughout the time of observation consistent with the $\overrightarrow{0}$ diagnosis of Clutton joints. A bone scan was not done. All 11 patients described by Clutton had bilateral hydro- $\vec{\omega}$ arthrosis, bilateral keratitis and 4 had osteitis of the tibia $^{3}$. Usually each case has two or more signs compatible with congenital syphilis such as Hutchinson teeth, eighth nerve deafness, keratitis and periostitis ${ }^{6} . \vec{\omega}$ One case report had a negative syphilis serology at or presentation but the diagnosis of SJD was made from the family history, positive serology in the mother and $\overrightarrow{\vec{T}}$ typical congenital clinical features ${ }^{7}$. In the absence of: a) $\vec{z}$ Signs of congenital syphilis in the patient discussed an b) Syphilis serology in the mother, (father deceased $\overrightarrow{\$} \vec{c}$ the diagnosis of Clutton joints is only a probability. SJE may be confused with rheumatoid arthritis but bo diseases may co-exist ${ }^{8}$. SJD affects men more tho $\ddot{\emptyset}_{0}$ women, larger joints, distal intephalangeal joints, arg $\bullet$ responds to anti-syphilitic therapy. One should be awa of a false positive Syphilis serology but with the presen of improved sensitive tests such as immunofluoresce trepone antibody test, the diagnosis is not likely to be missed. False positive rheumatoid factor tests have also $\stackrel{\square}{\mathbb{Q}}$ been described in secondary syphilis ${ }^{9}$. Some viral $\underset{\vec{P}}{\vec{P}}$ infections such as hepatitis B can cause joint symptoms. $\stackrel{\circ}{3}$ Non syphilitic treponematosis may cause difficulty in diagnosis as there are no serological tests to distinguish syphilis, yaws or pinta. Pinta is restricted to Central and South America and does not affect bone. The most significant sign of previous yaws and pinta infection is atrophic depigmented scars. The patient discussed has no such scars. There is no report of yaws presenting as symmetrical joint effusion.

Nearly all cases described in the literature responded well to anti-syphilitic treatment, usually intramuscular 3 . penicillin given in higher doses whenever the joint $\delta$ symptoms were chronic. There were no relapses and in some cases there was sero-conversion after therapy. A 윽 patient with left knee effusion developed a red-hot $>$ swollen knee and interstitial keratitis during the fourth week of intramuscular penicillin therapy and had to be $\bar{N}$ treated for a total of ten weeks with no dramaticos improvement ${ }^{6}$. After aspiration of the bilateral knee $\mathbb{O}$ effusions, our patient was given a course of oral N్ erythromycin but the effusions recurred three months 
Table 1

Differential Diagnosis of Syphilitic Joint Disease (Clutton Joints)

\begin{tabular}{ll}
\hline Joint Disease with Effusion & \multicolumn{1}{c}{ Comments } \\
\hline Rheumatoid & $\begin{array}{l}\text { Peripheral joints initially affected, } \\
\text { destructive process, more common } \\
\text { in women. }\end{array}$ \\
\hline Traumatic & Rarely bilateral. \\
\hline $\begin{array}{l}\text { Gonococcal } \\
\text { Tuberculosis }\end{array}$ & $\begin{array}{l}\text { Symptoms and signs of acute } \\
\text { inflammation, destructive. }\end{array}$ \\
\hline $\begin{array}{l}\text { Typhoid } \\
\text { Meningococcal }\end{array}$ & $\begin{array}{l}\text { Constitutional symptoms and signs } \\
\text { septicaemic illness, rash. }\end{array}$ \\
\hline Pyogenic & Usually monoarthritis, destructive \\
\hline Rheumatic Fever & May mimic secondary syphilis. \\
\hline $\begin{array}{l}\text { Systemic Lupus } \\
\text { Erythematosis }\end{array}$ & Multisystem involvement. \\
\hline Auto-immune Connective & Multisystem involvement. \\
Disorder & Other organ involvement. \\
\hline Sarcoidosis & $\begin{array}{l}\text { Usually young adult men, } \\
\text { conjunctivitis. }\end{array}$ \\
\hline Reiter's Disease &
\end{tabular}

afterwards. The right knee was re-aspirated and intraarticular steroid given. There is only one case report of penicillin and intra-articular steroids having been used in $\mathrm{SJD}^{10}$. Anandam gave oral steroids with penicillin ${ }^{7}$. Anti-syphilitic treatment on its own is usually sufficient. Some patients have had deterioration of symptoms with increasing blindness and deafness despite successful joint response to anti-syphilitic therapy ${ }^{4}$. Therefore although this patient had no symptoms or signs suggestive of congenital syphilis, she needs long term follow-up of her vision and hearing.

\section{Acknowledgement}

The author is grateful to Colonel G O Cowan who read the manuscript, Mrs M Hutton for her secretarial ?. assistance and the Librarians of the RAM College Millbank for literature search.

\section{REFERENCES}

1. Chandrasekaran A N et al. Sero Reactivity to Syphilis in $\vec{\nabla}$ Joint Disease. J Assoc Physicians India 1979; 27: Part 5 445-51.

2. Syphilitic Arthritis-JAMA 1973; 224: No 5 Section 37755.

3. ClutTon H H. Symmetrical Synovitis of Knee in Hereditary Syphilis. Lancet 1886; 1: 391-393.

4. SCOTT GRaY M and PhILP T. Syphilitic Arthritis, $\stackrel{\omega}{\sigma}$ Diagnostic Problems with special reference to Congenital Syphilis. Ann Rheum Dis 1963; 22: 19-25.

5. Reginato A J, et al. Synovitis in Secondary Syphilis, Arthritis and Rheumatism Vol 22 No 2 February 1979 170- $\vec{\omega}$ 176.

6. ARgEN R J and Dixon A J. Clutton's joints with Keratitis N and Periostitis, Arthritis and Rheumatism Vol 6 No 41963 341-47.

7. ANANDAM K, SRIJAYA R and Bhaskarala K P V. Cluttog 윽 Joints (A Case Report) . Indian J Dermatol, Vener a $\overline{\text { d }}$ Leprol 1979; 45: Part 2 139-142.

8. ChOPRA A and TUtakne M. Syphilis and Rheumat of Arthritis. J Assoc Physicians India 1986; 34: Part 4, 272요

9. Domonkos A N, Arnold H L Jr and Andrews O R Diseases of Skin. Toronto W B Saunders Co $1982467-468$

10. RoDin P: Clutton's Joints. A Brief Review of the literattooe and an unusual case treated with intra-articuor Hydrocortisone. Br J Vener Dis 1961; 37: 204. 